\title{
Article
}

\section{Oscillation of solutions to fourth-order delay differential equations with middle term}

\author{
Elmetwally M. Elabbasy ${ }^{1}$, Ethiraju Thandapani ${ }^{2}$, Osama Moaaz $^{1}$ and Omar Bazighifan ${ }^{3, *}$ \\ 1 Department of Mathematics, Faculty of Science, Mansoura University, 35516 Mansoura, Egypt.; \\ emelabbasy@mans.edu.eg(E.M.E); o.moaaz@mans.edu.eg(O.M) \\ 2 University of Madras Ramanujan Institute for Advanced Study in Mathematics Chennai, 600005, India.; \\ ethandapani@yahoo.co.in \\ 3 Department of Mathematics; Faculty of Science; Hadhramout University; Hadhramout 50512, Yemen. \\ * Correspondence: o.bazighifan@gmail.com
}

Received: 12 December 2018; Accepted: 11 March 2019; Published: 01 Jun 2019.

\begin{abstract}
This work is concerned with the oscillatory behavior of fourth-order delay differential equation with middle term. By using the generalized Riccati transformations and new comparison principles, we establish new oscillation results for this equation. An example illustrating the results is also given.
\end{abstract}

Keywords: Fourth-order, differential equations, oscillatory solutions, technique of Riccati transformation, comparison theorem.

MSC: 34K10, 34K11.

\section{Introduction}

I

$\mathrm{n}$ this work, we study asymptotic properties of solutions of differential equation of the fourth order and with middle term

$$
\left(r(\ell) y^{\prime \prime \prime}(\ell)\right)^{\prime}+p(\ell) y^{\prime \prime \prime}(\ell)+q(\ell) y(\sigma(\ell))=0, \quad \ell \geq \ell_{0} .
$$

We assume $r(\ell) \in C\left(\left[\ell_{0}, \infty\right), \mathbb{R}\right), r(\ell)>0$ for all $\ell \geq \ell_{0}, p, q, \sigma \in C\left(\left[\ell_{0}, \infty\right), \mathbb{R}\right)$ such that $p(\ell) \geq 0, q>$ $0, \sigma(\ell) \leq \ell$ and $\lim _{\ell \rightarrow \infty} \sigma(\ell)=\infty$. We say that a function $y(\ell)$ is a solution of (1), we mean a non-trivial real function $y(\ell) \in C\left(\left[\ell_{y}, \infty\right)\right), \ell_{y} \geq \ell_{0}$, satisfying (1) on $\left[\ell_{y}, \infty\right)$ and moreover, having the properties: $y(\ell), y^{\prime}(\ell)$, $y^{\prime \prime}(\ell)$ and $r(\ell)\left[y^{\prime \prime \prime}(\ell)\right]$ are continuously differentiable for all $\ell \in\left[\ell_{y}, \infty\right)$. We consider only those solutions $y(\ell)$ of (1) which satisfy $\sup \{|y(\ell)|: \ell \geq \ell\}>0$, for any $\ell \geq \ell_{y}$. A solution of (1) is called oscillatory if it has arbitrary large zeros, otherwise it is called nonoscillatory.

The oscillations of higher and fourth order differential equations have been studied by several authors and several techniques have been proposed for obtaining oscillatory criteria for higher and fourth order differential equations. For treatments on this subject, we refer the reader to the texts [1-6] and the articles [7-25]. In what follows, we review some results that have provided the background and the motivation, for the present work.

Hou and Cheng [22] studied the oscillation of differential equation with middle term

$$
y^{(4)}(\ell)+p(\ell) y^{\prime}(\ell)+q(\ell) f(y(\sigma(\ell)))=0, \quad \ell \geq \ell_{0},
$$

under the condition: third-order differential equation $z^{\prime \prime \prime}(\ell)+p(\ell) z(\ell)=0$, is nonoscillatory.

Džurina and Jadlovskă [11] have considered the differential equation of the fourth order with a negative term

$$
y^{(4)}(\ell)+p(\ell) y^{\prime}(\ell)+q(\ell) y(\sigma(\ell))=0, \quad \ell \geq \ell_{0}
$$

under the condition: all solutions of the contributory third-order differential equation are nonoscillatory. 
Moaaz et al. [19] studied the oscillatory behavior of the third-order nonlinear differential equation with middle term of the form

$$
\left[r_{2}(\ell)\left(r_{1}(\ell)\left(y^{\prime}(\ell)\right)^{\alpha}\right)^{\prime}\right]^{\prime}+\phi\left(\ell, y^{\prime}(\sigma(\ell))\right)+q(\ell) f(y(\sigma(\ell)))=0, \quad \ell \geq \ell_{0},
$$

under

$$
\int_{\ell_{0}}^{\infty} \frac{1}{r^{\frac{1}{\alpha}}(\ell)} d \ell=\infty
$$

Our aim, in the present work, is to use the generalized Riccati transformations and new comparison principles to obtain a new conditions for the oscillation of every solutions of (1) under condition

$$
R(\ell)=\int_{\ell_{0}}^{\ell} \frac{1}{r(s)} \exp \left(-\int_{\ell_{0}}^{s} \frac{p(u)}{r(u)} d u\right) d s \text { and } R(\ell) \rightarrow \infty \text { as } \ell \rightarrow \infty .
$$

An example is included to illustrate the main results.

\section{Main results}

In the next section, we shall establish some oscillation criteria for equation (1). The proof of our main results are essentially based on the following lemmas.

Lemma 1. (See [20], Lemma 1) Let $\beta \geq 1$ be a ratio of two numbers, where $U$ and $V$ are constants. Then

$$
U z-V z^{\frac{\beta+1}{\beta}} \leq \frac{\beta^{\beta}}{(\beta+1)^{\beta+1}} \frac{U^{\beta+1}}{V^{\beta}}, V>0 .
$$

Lemma 2. (See [6], Lemma 13) If the function $u$ satisfies $u^{(\kappa)}>0, \kappa=0,1, \ldots, m$, and $u^{(m+1)}<0$, then

$$
\frac{u(\ell)}{\ell^{m} / m !} \geq \frac{u^{\prime}(\ell)}{\ell^{m-1} /(m-1) !}
$$

Lemma 3. (See [1], Lemma 2.2.3) Let $h \in C^{n}\left(\left[\ell_{0}, \infty\right),(0, \infty)\right)$. Let $h^{(n)}(\ell)$ is of a fixed sign and on the interval $\left[\ell_{0}, \infty\right), h^{(n)}(\ell)$ not identically zero, and that there exists a $\ell_{1} \geq \ell_{0}$ such that $h^{(n-1)}(\ell) h^{(n)}(\ell) \leq 0$ for all $\ell \geq \ell_{1}$. If $\lim _{\ell \rightarrow \infty} h(\ell) \neq 0$ then for every $\lambda \in(0,1)$ there exists $\ell_{\lambda} \geq \ell_{0}$ such that

$$
h(\ell) \geq \frac{\lambda}{(n-1) !} \ell^{n-1}\left|h^{(n-1)}(\ell)\right| \text { for } \ell \geq \ell_{\lambda}
$$

For convenience, we denote

$$
\rho_{+}^{\prime}(\ell):=\max \left\{0, \rho_{+}^{\prime}(\ell)\right\} \text { and } \delta_{+}^{\prime}(\ell):=\max \left\{0, \delta^{\prime}(\ell)\right\} .
$$

Theorem 4. Assume that (3) holds. If there exist positive functions $\rho, \delta \in C^{1}\left(\left[\ell_{0}, \infty\right)\right)$ such that

$$
\int_{\ell_{0}}^{\infty}\left(\rho(s) q(s) \frac{\mu}{2} \sigma^{2}(s)-\frac{1}{4 \rho(s) r(s)}\left[\frac{\rho_{+}^{\prime}(s)}{\rho(s)}-\frac{p(s)}{r(s)}\right]^{2}\right) d s=\infty,
$$

for some $\mu \in(0,1)$, and

$$
\int_{\ell_{0}}^{\infty}\left[\delta(s) \int_{s}^{\infty}\left[\frac{1}{r(v)} \int_{v}^{\infty} q(v)\left(\frac{\sigma^{2}(v)}{v^{2}}\right) d v\right] d v-\frac{\left(\delta^{\prime}(s)\right)^{2}}{4 \delta(s)}\right] d s=\infty,
$$

then all solutions of (1) are oscillatory. 
Proof. Assume that (1) has a solution $y$ is nonoscillatory. Without loss of generality, we may assume that there exists a $\ell_{1} \in\left[\ell_{0}, \infty\right)$ such that $y(\ell)>0, y(\sigma(\ell))>0$ for all $\ell \in\left[\ell_{0}, \infty\right)$. It follows from (1) and (3) that $\left(r(\ell) y^{\prime \prime \prime}(\ell)\right)^{\prime}<0$ and there exist two possible cases for $\ell \geq \ell_{1}$, where $\ell_{1} \geq \ell_{0}$ is sufficiently large:

$$
\begin{aligned}
& \left(C_{1}\right) y^{(\kappa)}(\ell)>0 \text { for } \kappa=0,1,2,3 \\
& \left(C_{2}\right) y^{(\kappa)}(\ell)>0 \text { for } \kappa=0,1,3 \text {, and } y^{\prime \prime}(\ell)<0 .
\end{aligned}
$$

Assume that we have Case $\left(C_{1}\right)$. We define a generalized Riccati substitution by

$$
\omega(\ell):=\rho(\ell) \frac{r(\ell)\left(y^{\prime \prime \prime}(\ell)\right)}{y^{\prime \prime}} .
$$

Then $\omega(\ell)>0$. Differentiating, we obtain

$$
\begin{aligned}
\omega^{\prime}(\ell) & =\rho(\ell)\left(\frac{r(\ell)\left(y^{\prime \prime \prime}(\ell)\right)}{y^{\prime \prime}}\right)^{\prime}+\rho^{\prime}(\ell) \frac{r(\ell)\left(y^{\prime \prime \prime}(\ell)\right)}{y^{\prime \prime}(\ell)}, \\
& =\rho(\ell) \frac{\left(r(\ell)\left(y^{\prime \prime \prime}(\ell)\right)\right)^{\prime}}{y^{\prime \prime}}-\rho(\ell) \frac{r(\ell)\left(y^{\prime \prime \prime}(\ell)\right)^{2}}{\left(y^{\prime \prime}\right)^{2}}+\rho^{\prime}(\ell) \frac{r(\ell)\left(y^{\prime \prime \prime}(\ell)\right)}{y^{\prime \prime}} .
\end{aligned}
$$

By virtue of (1), we have.

$$
\left(r(\ell) y^{\prime \prime \prime}(\ell)\right)^{\prime}+p(\ell) y^{\prime \prime \prime}(\ell)=-q(\ell) y(\sigma(\ell))
$$

From (7),we see that

$$
\omega^{\prime}(\ell) \leq \rho_{+}^{\prime}(\ell) \frac{r(\ell)\left(y^{\prime \prime \prime}(\ell)\right)}{y^{\prime \prime}}-\rho(\ell) \frac{p(\ell) y^{\prime \prime \prime}(\ell)}{y^{\prime \prime}}-\frac{\rho(\ell)(q(\ell) y(\sigma(\ell)))}{y^{\prime \prime}}-\rho(\ell) \frac{r(\ell)\left(y^{\prime \prime \prime}(\ell)\right)^{2}}{\left(y^{\prime \prime}\right)^{2}} .
$$

Hence, by (7), we obtain

$$
\omega^{\prime}(\ell) \leq \frac{\rho_{+}^{\prime}(\ell)}{\rho(\ell)} \omega(\ell)-\frac{p(\ell)}{r(\ell)} \omega(\ell)-\rho(\ell) q(\ell) \frac{y(\sigma(\ell))}{y^{\prime \prime}}-\frac{1}{\rho(\ell) r(\ell)} \omega(\ell)^{2} .
$$

From Lemma 2, we have that $y(\ell) \geq \frac{\ell}{2} y^{\prime}(\ell)$ and hence,

$$
\frac{y^{\prime}(\ell)}{y(\ell)} \leq \frac{2}{\ell}
$$

Integrating from $\ell$ to $\sigma(\ell)$ we find

$$
\frac{y(\sigma(\ell))}{y(\ell)} \geq \frac{\sigma^{2}(\ell)}{\ell^{2}}
$$

This implies that

$$
y(\sigma(\ell)) \geq \frac{\sigma^{2}(\ell)}{\ell^{2}} y(\ell)
$$

It follows from Lemma 3 that

$$
y(\ell) \geq \frac{\mu}{2} \ell^{2} y^{\prime \prime}(\ell)
$$

for all $\mu \in(0,1)$ and every sufficiently large $\ell$. Thus, by (8), (9) and (10), we get

$$
\begin{aligned}
\omega^{\prime}(\ell) & \leq \frac{\rho_{+}^{\prime}(\ell)}{\rho(\ell)} \omega(\ell)-\frac{p(\ell)}{r(\ell)} \omega(\ell)-\rho(\ell) q(\ell) \frac{\sigma^{2}(\ell)}{\ell^{2}} \frac{y(\ell)}{y^{\prime \prime}}-\frac{1}{\rho(\ell) r(\ell)} \omega(\ell)^{2}, \\
& \leq \frac{\rho_{+}^{\prime}(\ell)}{\rho(\ell)} \omega(\ell)-\frac{p(\ell)}{r(\ell)} \omega(\ell)-\rho(\ell) q(\ell)\left(\frac{\sigma(\ell)}{\ell}\right)^{2} \frac{\mu}{2} \ell^{2}-\frac{1}{\rho(\ell) r(\ell)} \omega(\ell)^{2} .
\end{aligned}
$$

This implies that

$$
\omega^{\prime}(\ell) \leq\left[\frac{\rho_{+}^{\prime}(\ell)}{\rho(\ell)}-\frac{p(\ell)}{r(\ell)}\right] \omega(\ell)-\rho(\ell) q(\ell) \frac{\mu}{2} \sigma^{2}(\ell)-\frac{1}{\rho(\ell) r(\ell)} \omega(\ell)^{2} .
$$


Using Lemma 1 with $U=\left[\frac{\rho_{+}^{\prime}(\ell)}{\rho(\ell)}-\frac{p(\ell)}{r(\ell)}\right], V=\frac{1}{\rho(\ell) r(\ell)}$ and $z=\omega$, we get

$$
\left[\frac{\rho_{+}^{\prime}(\ell)}{\rho(\ell)}-\frac{p(\ell)}{r(\ell)}\right] \omega(\ell)-\frac{1}{\rho(\ell) r(\ell)} \omega(\ell)^{2} \leq \frac{1}{4 \rho(\ell) r(\ell)}\left[\frac{\rho_{+}^{\prime}(\ell)}{\rho(\ell)}-\frac{p(\ell)}{r(\ell)}\right]^{2} .
$$

Hence, we obtain

$$
\omega^{\prime}(\ell) \leq-\rho(\ell) q(\ell) \frac{\mu}{2} \sigma^{2}(\ell)+\frac{1}{4 \rho(\ell) r(\ell)}\left[\frac{\rho_{+}^{\prime}(\ell)}{\rho(\ell)}-\frac{p(\ell)}{r(\ell)}\right]^{2} .
$$

Which implies that

$$
\int_{\ell_{1}}^{\ell}\left(\rho(s) q(s) \frac{\mu}{2} \sigma^{2}(s)-\frac{1}{4 \rho(s) r(s)}\left[\frac{\rho_{+}^{\prime}(s)}{\rho(s)}-\frac{p(s)}{r(s)}\right]^{2}\right) d s \leq \omega\left(\ell_{1}\right),
$$

for some $\mu \in(0,1)$ which contradicts (4).

Assume that we have Case $\left(C_{2}\right)$ holds. Define

$$
\psi(\ell):=\delta(\ell) \frac{y^{\prime}(\ell)}{y(\ell)}, \ell \geq \ell_{1} .
$$

Then $\psi(\ell)>0$ for $\ell \geq \ell_{1}$ and

$$
\begin{aligned}
\psi^{\prime}(\ell) & =\delta^{\prime}(\ell) \frac{y^{\prime}(\ell)}{y(\ell)}-\delta(\ell) \frac{y^{\prime \prime}(\ell) y(\ell)-\left(y^{\prime}\right)^{2}(\ell)}{y^{2}(\ell)}, \\
& =\delta(\ell) \frac{y^{\prime \prime}(\ell)}{y(\ell)}+\frac{\delta^{\prime}(\ell)}{\delta(\ell)} \psi(\ell)-\frac{\psi^{2}(\ell)}{\delta(\ell)} .
\end{aligned}
$$

Integrating (1) from $\ell$ to $u$ we find

$$
r(u)\left(y^{\prime \prime \prime}\right)(u)-r(\ell)\left(y^{\prime \prime \prime}\right)(\ell)+\int_{\ell}^{u} q(s) y(\sigma(s)) d s \leq 0 .
$$

By virtue of $y^{\prime}(\ell)>0, y(\ell)>0$ and $y^{\prime \prime}(\ell)<0$ by (9), we obtain

$$
r(u)\left(y^{\prime \prime \prime}\right)(u)-r(\ell)\left(y^{\prime \prime \prime}\right)(\ell)+\int_{\ell}^{u} q(s)\left(\frac{\sigma^{2}(s)}{s^{2}}\right) y(\ell) d s \leq 0,
$$

whence it follows, by $y^{\prime}(\ell)>0$, that

$$
r(u)\left(y^{\prime \prime \prime}\right)(u)-r(\ell)\left(y^{\prime \prime \prime}\right)(\ell)+y(\ell) \int_{\ell}^{u} q(s)\left(\frac{\sigma^{2}(s)}{s^{2}}\right) d s \leq 0 .
$$

Letting $u \rightarrow \infty$, we arrive at the inequality

$$
-r(\ell)\left(y^{\prime \prime \prime}\right)(\ell)+y(\ell) \int_{\ell}^{\infty} q(s)\left(\frac{\sigma^{2}(s)}{s^{2}}\right) d s \leq 0,
$$

we get

$$
y^{\prime \prime}(\ell)+y(\ell) \int_{\ell}^{\infty}\left[\frac{1}{r(\ell)} \int_{v}^{\infty} q(\ell)\left(\frac{\sigma^{2}(s)}{s^{2}}\right) d s\right] d v \leq 0,
$$

we see

$$
\frac{y^{\prime \prime}(\ell)}{y(\ell)} \leq-\left[\frac{1}{r(\ell)} \int_{v}^{\infty} q(\ell)\left(\frac{\sigma^{2}(s)}{s^{2}}\right) d s\right] d v .
$$

Hence, by (16) in (14), we find

$$
\psi^{\prime}(\ell) \leq-\delta(\ell) \int_{\ell}^{\infty}\left[\frac{1}{r(v)} \int_{v}^{\infty} q(\ell)\left(\frac{\sigma^{2}(s)}{s^{2}}\right) d s\right] d v+\frac{\delta^{\prime}(\ell)}{\delta(\ell)} \psi(\ell)-\frac{\psi^{2}(\ell)}{\delta(\ell)} .
$$


Thus, we have

$$
\psi^{\prime}(\ell) \leq-\delta(\ell) \int_{\ell}^{\infty}\left[\frac{1}{r(v)} \int_{v}^{\infty} q(s)\left(\frac{\sigma^{2}(s)}{s^{2}}\right) d s\right] d v+\frac{\left(\delta^{\prime}(\ell)\right)^{2}}{4 \delta(\ell)}
$$

Integrating from $\ell_{1}$ to $\ell$, we get

$$
\int_{\ell_{1}}^{\ell}\left[\delta(s) \int_{s}^{\infty}\left[\frac{1}{r(v)} \int_{v}^{\infty} q(v)\left(\frac{\sigma^{2}(v)}{v^{2}}\right) d v\right] d v-\frac{\left(\delta^{\prime}(s)\right)^{2}}{4 \delta(s)}\right] d s \leq \psi\left(\ell_{1}\right)
$$

which contradicts (5).

The proof of the theorem is complete.

Another criteria for oscillation of (1) can be establish by comparison with ordinary equations of the lower order. We extend a comparison theorem that fasten properties of solutions of (1) with those of second-order differential equations. It is well known (see [1]) that the differential equation

$$
\left[a(\ell)\left(y^{\prime}(\ell)\right)\right]^{\prime}+q(\ell) y(\ell)=0, \quad \ell \geq \ell_{0}
$$

where $a, q \in C\left[\ell_{0}, \infty\right), a(\ell), q(\ell)>0$, and the necessary and sufficient condition for nonoscillatory of this equation is to there exist a number $\ell \geq \ell_{0}$, and a function $v \in C^{1}[\ell, \infty)$, satisfying

$$
v^{\prime}(\ell)+a^{-1}(\ell) v^{2}(\ell)+q(\ell) \leq 0, \quad \text { on }[\ell, \infty) .
$$

In the following, we compare the behavior of oscillatory of (1) with the half-linear differential equations of type (19).

Lemma 5. (see[1]) Let

$$
\int_{\ell_{0}}^{\infty} \frac{1}{a(s)} d s=\infty
$$

Then the condition

$$
\liminf _{\ell \rightarrow \infty}\left(\int_{\ell_{0}}^{\infty} \frac{1}{a(s)} d s\right) \int_{\ell}^{\infty} q(s) d s>\frac{1}{4}
$$

guarantees oscillation of (19).

Theorem 6. Let (3) and assume that the equation

$$
\left[r(\ell) y^{\prime}(\ell)\right]^{\prime}+q(\ell) \frac{\mu}{2} \sigma^{2}(\ell) y(\ell)=0,
$$

and

$$
y^{\prime \prime}(\ell)+\left(\int_{\ell}^{\infty}\left[\frac{1}{r(v)} \int_{v}^{\infty} q(s)\left(\frac{\sigma^{2}(s)}{s^{2}}\right) d s\right] d v\right) y(\ell)=0
$$

are oscillatory, then every solution of (1) is oscillatory.

Proof. Proceeding as in proof of the Theorem 4. If we set $\rho(\ell)=1$ in (11), then we get

$$
\omega^{\prime}(\ell)+\frac{p(\ell)}{r(\ell)} \omega(\ell)+q(\ell) \frac{\mu}{2} \sigma^{2}(\ell)+\frac{1}{r(\ell)} \omega(\ell)^{2} \leq 0,
$$

for every constant $\mu \in(0,1)$. Thus, we can see that equation (20) is nonoscillatory for every constant $\mu \in$ $(0,1)$, which is a contradiction. If we now set $\delta(\ell)=1$ in $(17)$, then we find

$$
\psi^{\prime}(\ell)+\int_{\ell}^{\infty}\left[\frac{1}{r(v)} \int_{v}^{\infty} q(s)\left(\frac{\sigma^{2}(s)}{s^{2}}\right) d s\right] d v+\psi^{2}(\ell) \leq 0
$$

Hence, equation (21) is nonoscillatory, which is a contradiction.

Theorem 6 is proved. 


\section{Example}

In this section, we give the following example to illustrate our main results.

Example 1. Consider the differential equation

$$
\left(\frac{1}{\ell} y^{\prime \prime \prime}(\ell)\right)^{\prime}+\left(1 \backslash 2 \ell^{2}\right) y^{\prime \prime \prime}(\ell)+\frac{\beta}{\ell} y\left(\frac{\ell}{2}\right)=0, \ell \geq 1,
$$

where $\beta>0$ is a constant. Let

$$
r(\ell)=\frac{1}{\ell}, p(\ell)=1 \backslash 2 \ell^{2}, \sigma(\ell)=\frac{\ell}{2}, p(\ell)=1 \backslash 2 \ell^{2}, q(\ell)=\frac{\beta}{\ell},
$$

we get

$$
R(\ell)=2 \backslash 3\left(\ell^{3 \backslash 2}-1\right) \rightarrow \infty \text { as } \ell \rightarrow \infty .
$$

If we now set $\rho(s)=\delta(s)=1$ then

$$
\begin{aligned}
& \int_{\ell_{0}}^{\infty}\left(\rho(s) q(s) \frac{\mu}{2} \sigma^{2}(s)-\frac{1}{4 \rho(s) r(s)}\left[\frac{\rho_{+}^{\prime}(s)}{\rho(s)}-\frac{p(s)}{r(s)}\right]^{2}\right) d s, \\
= & \left(\frac{\beta \mu}{8}-\frac{1}{4}\right) \int_{\ell_{0}}^{\infty} \ell d \ell=\infty, \text { if } \beta>\frac{2}{\mu} \text { for some constant } \mu \in(0,1)
\end{aligned}
$$

and

$$
\int_{\ell_{0}}^{\infty}\left[\delta(s) \int_{s}^{\infty}\left[\frac{1}{r(v)} \int_{v}^{\infty} q(v)\left(\frac{\sigma^{2}(v)}{v^{2}}\right) d v\right] d v-\frac{\left(\delta^{\prime}(s)\right)^{2}}{4 \delta(s)}\right] d s=\infty .
$$

Thus, by Theorem 4, every solution of equation (22) is oscillatory, provided $\beta>\frac{2}{\mu}$.

\section{Conclusion}

In this work, by using the generalized Riccati transformations technique and new comparison principles, we offer some new sufficient conditions which ensure that any solution of Equation (1) oscillates under the condition (3). Further, we can try to get some oscillation criteria of Equation (1) if $y(\ell)=x(\ell)+p(\ell) x(\tau(\ell))$ in the future work.

Acknowledgments: The authors thank the reviewers for for their useful comments, which led to the improvement of the content of the paper.

Author Contributions: All authors contributed equally to the writing of this paper. All authors read and approved the final manuscript.

Conflicts of Interest: The authors declare no conflict of interest.

\section{References}

[1] Agarwal, R. P., Grace, S. R., \& O'Regan, D. (2013). Oscillation theory for difference and functional differential equations. Springer Science \& Business Media.

[2] Bazighifan, O., Elabbasy, E. M., \& Moaaz, O. (2019). Oscillation of higher-order differential equations with distributed delay. Journal of Inequalities and Applications, 55(1), 1-9.

[3] Moaaz, O., Elabbasy, E. M., \& Bazighifan, O. (2017). On the asymptotic behavior of fourth-order functional differential equations. Advances in Difference Equations, 2017(1), 261.

[4] Györi, I., \& Ladas, G. E. (1991). Oscillation theory of delay differential equations: With applications. Oxford University Press, USA.

[5] Ladde, G. S., Lakshmikantham, V., \& Zhang, B. G. (1987). Oscillation theory of differential equations with deviating arguments (Vol. 110). Marcel Dekker Incorporated.

[6] Kiguradze, I., \& Chanturia, T. A. (2012). Asymptotic properties of solutions of nonautonomous ordinary differential equations (Vol. 89). Springer Science \& Business Media.

[7] Agarwal, R. P., Grace, S. R., \& Wong, P. J. (2005). On the bounded oscillation of certain fourth order functional differential equations. Nonlinear Dynamics and Systems Theory, 5, 215-227. 
[8] Agarwal, R. P., Bohner, M., Li, T., \& Zhang, C. (2014). Oscillation theorems for fourth-order half-linear delay dynamic equations with damping. Mediterranean Journal of Mathematics, 11(2), 463-475.

[9] Dzurina, J., Baculikova, B., \& Jadlovska, I. (2015). Oscillation of solutions to fourth-order trinomial delay differential equations. Electronic Journal of Differential Equations, 2015(70), 1-10.

[10] Bazighifan, O. (2017). Oscillatory behavior of higher-order delay differential equations. General Letters in Mathematics, 2, 105-110.

[11] Džurina, J., \& Jadlovská, I. (2017). Oscillation theorems for fourth-order delay differential equations with a negative middle term. Mathematical Methods in the Applied Sciences, 40(18), 7830-7842.

[12] Dong, J. G. (2010). Oscillation behavior of second order nonlinear neutral differential equations with deviating arguments. Computers $\mathcal{E}$ Mathematics with Applications, 59(12), 3710-3717.

[13] Chen, D. X. (2010). Oscillation and asymptotic behavior for nth-order nonlinear neutral delay dynamic equations on time scales. Acta Applicandae Mathematicae, 109(3), 703-719.

[14] Elabbasy, E. M., Moaaz, O., \& Bazighifan, O. (2017). Oscillation criteria for fourth-order nonlinear differential equations. International Journal of Modern Mathematical Sciences, 15(1), 50-57.

[15] Grace, S. R., \& Lalli, B. S. (1984). Oscillation theorems for nth order nonlinear differential equations with deviating arguments. Proceedings of the American Mathematical Society, 65-70.

[16] Grace, S. R., Agarwal, R. P., \& Graef, J. R. (2009). Oscillation theorems for fourth order functional differential equations. Journal of Applied Mathematics and Computing, 30(1-2), 75-88.

[17] Cesarano, C., Pinelas, S., Al-Showaikh, F., \& Bazighifan, O. (2019). Asymptotic Properties of Solutions of Fourth-Order Delay Differential Dquations. Symmetry, 11(5), 628.

[18] Cesarano, C., \& Bazighifan, O. (2019). Oscillation of fourth-order functional differential equations with distributed delay. Axioms, 8(2), 61.

[19] Moaaz, O., Elabbasy, E. M., \& Shaaban, E. (2018). Oscillation criteria for a class of third order damped differential equations. Arab Journal of Mathematical Sciences, 24(1), 16-30.

[20] Tunc, C. E. M. I. L., \& Bazighifan, O. M. A. R. (2019). Some new oscillation criteria for fourth-order neutral differential equations with distributed delay. Electron. J. Math. Anal. Appl, 7, 235-241.

[21] Philos, C. G. (1981). On the existence of nonoscillatory solutions tending to zero at8 for differential equations with positive delays. Archiv der Mathematik, 36(1), 168-178.

[22] Hou, C., \& Cheng, S. S. (2009). Asymptotic dichotomy in a class of fourth-order nonlinear delay differential equations with damping. In Abstract and Applied Analysis (Vol. 2009). Hindawi.

[23] Zhang, C., Li, T., Agarwal, R. P., \& Bohner, M. (2012). Oscillation results for fourth-order nonlinear dynamic equations. Applied Mathematics Letters, 25(12), 2058-2065.

[24] Zhang, C., Li, T., Sun, B., \& Thandapani, E. (2011). On the oscillation of higher-order half-linear delay differential equations. Applied Mathematics Letters, 24(9), 1618-1621.

[25] Zhang, C., Li, T., \& Saker, S. H. (2014). Oscillation of fourth-order delay differential equations. Journal of Mathematical Sciences, 201(3), 296-309. 